Document downloaded from:

http://hdl.handle.net/10251/44271

This paper must be cited as:

García Hernández, J.; Heras Barberá, SM.; Juan Císcar, A.; Paredes Palacios, R.; Nácher Rodríguez, B.; Alemany, S.; Alcántara, E.... (2005). The MORFO3D foot database. En Pattern Recognition and Image Analysis: Second Iberian Conference, IbPRIA 2005, Estoril, Portugal, June 7-9, 2005, Proceedings, Part II. Springer Verlag (Germany). 658665. doi:10.1007/11492542_80.

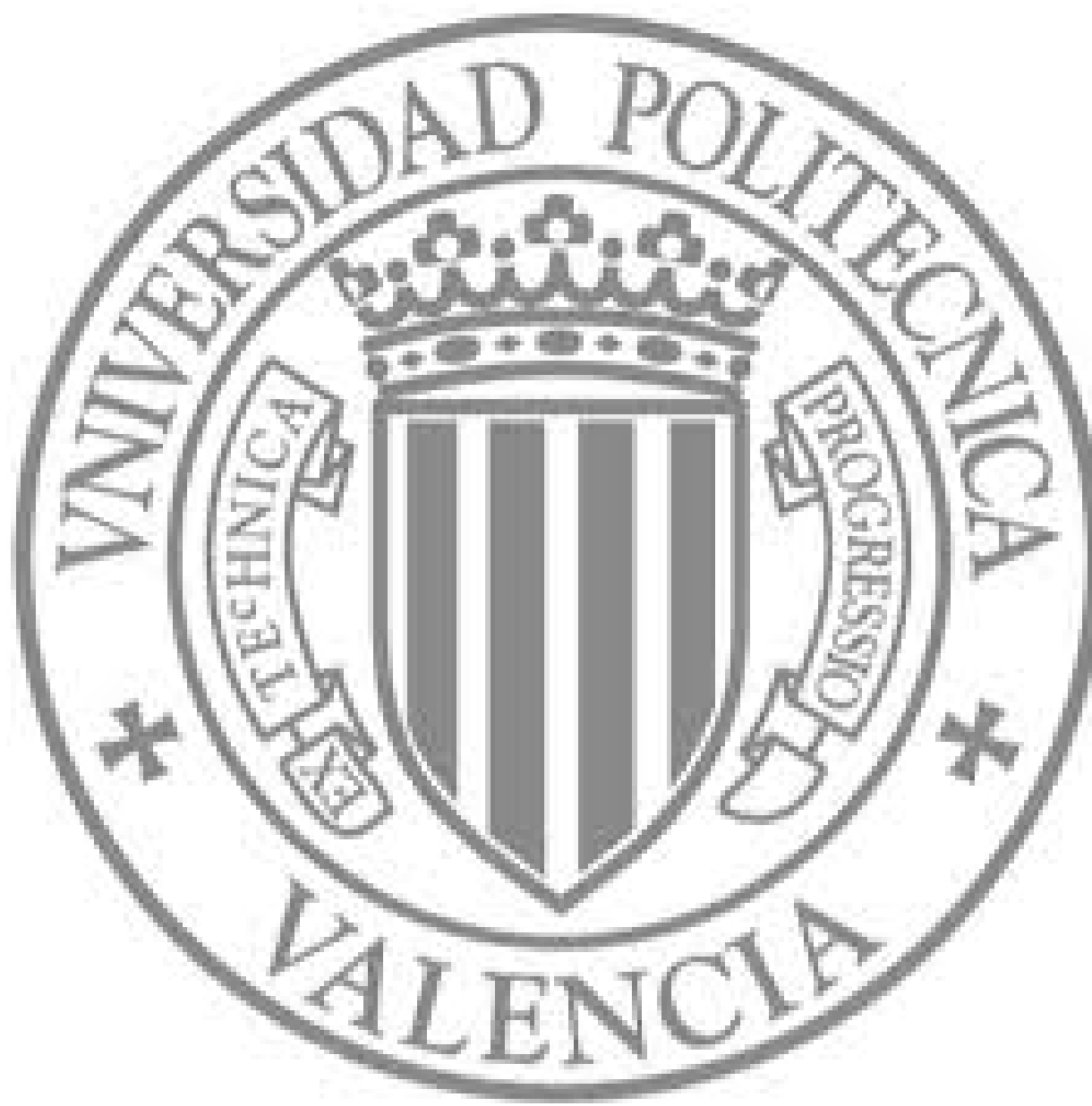

The final publication is available at

http://link.springer.com/chapter/10.1007/11492542_80

Copyright

Springer Verlag (Germany) 


\title{
The MORFO3D foot database ${ }^{\star}$
}

\author{
José García-Hernández ${ }^{1}$, Stella Heras ${ }^{1}$, Alfons Juan ${ }^{1}$, Roberto Paredes ${ }^{1}$, \\ Beatriz Nácher ${ }^{2}$, Sandra Alemany ${ }^{2}$, Enrique Alcántara ${ }^{2}$, and Juan Carlos \\ González ${ }^{2}$ \\ 1 Departament de Sistemes Informàtics i Computació \\ 2 Institut de Biomecànica de València \\ Universitat Politècnica de València \\ [jogarcia, sheras, ajuan, rparedes] @dsic.upv.es \\ [beanacfe, maalmut, ealcanta, jcgonza] @ibv.upv.es
}

\begin{abstract}
A foot database comprising 3D foot shapes and footwear fitting reports of more than 300 participants is presented. It was primarily acquired to study footwear fitting, though it can also be used to analyse anatomical features of the foot. In fact, we present a technique for automatic detection of several foot anatomical landmarks, together with some empirical results.
\end{abstract}

\section{Introduction}

Footwear fitting has a decisive influence on the functionality and comfort of shoes. The conventional technique to estimate footwear fitting is the direct try of shoes, which is perfectly possible in most cases. However, there are many cases in which it is not possible due to the amount of models to try, physical obstacles to the technique (e.g. in e-commerce), or physical or psychical problems related with the user (e.g. sensitivity difficulties). In all of these cases, automatic fitting prediction can help a lot.

In this work, we present a foot database developed in the Spanish research project MORFO3D. The MORFO3D foot database was acquired during the month of May 2004 at the Universitat Politècnica de València, València (Spain). It comprises 3D foot shapes and footwear fitting reports of more than 300 participants. The database was primarily acquired to study footwear fitting, though it can also be used to analyse anatomical features of the foot. In fact, we present a knowledge-based technique for automatic detection of several foot anatomical landmarks that gives good results. The MORFO3D foot database is available upon request for non-commercial use.

The acquisition of the MORFO3D foot database is described in section 2. Then, in section 3, we present our technique for automatic detection of several foot anatomical landmarks, together with some empirical results.

\footnotetext{
* Work supported by the "Agència Valenciana de Ciència i Tecnologia" under grant GRUPOS03/031 and the Spanish projects DPI2001-0880-CO2-01 and DPI20010880-CO2-02.
} 


\section{The MORFO3D foot database}

The MORFO3D foot database was acquired during the month of May 2004 at the Universitat Politècnica de València, València (Spain). A total of 316 18to 35-year-old women of European shoe size 38 (and 40) participated in its acquisition. It is considered that the feet of the women in this age interval is enough developed, but still not shows the typical advanced age pathologies. For each participant, we first acquired her right 3D foot shape together with the location of several foot key points (landmarks). Then, we asked her to try 4 models of shoes (out of 8 available) and fill in a questionnaire about each shoe fitting. Hereafter we provide a detailed description of these two basic acquisition steps.

For the acquisition of 3D foot shapes, we used an INFOOT laser scanner [1]. This scanner is able to acquire a complete $3 \mathrm{D}$ shape of the foot, including the foot sole. Also, it can be used to acquire the location of foot landmarks by simply marking them on the user barefoot with adhesive markers. In our case, the acquisition process is as illustrated in Figure 1. The process begins with the placement of adhesive markers on 14 foot landmarks located on bony prominences or critical zones for shoe fitting (see top of Figure 1 for their precise locations). Then, the scanning process is carried out while the participant stands upright with equal weight on each foot, in a certain position and orientation (see middle of Figure 1). The result consists of: a) a cloud of points representing the outer surface of the foot; b) the location of the 14 foot landmarks previously marked; and c) a number of podometric measurements derived from these 14 landmarks (see bottom of Figure 1). The complete acquisition process lasted approximately 5 minutes on average.

Table 1 shows some descriptive statistics of the 3D foot shapes acquired. The main differences among the feet measurements taken were observed in the height of the external malleolus and the foot length.

Table 1. Descriptive statistics (in $\mathrm{mm}$ ) of the $3 \mathrm{D}$ foot shapes acquired.

\begin{tabular}{ccccc} 
Podometric variable & Min & Mean & Max Standard deviation \\
\hline Foot length & 225.9 & 241.77 & 257.6 & \pm 5.82 \\
Forefoot width & 84.5 & 94.07 & 106.4 & \pm 3.95 \\
Hell width & 55.2 & 61.44 & 70.5 & \pm 2.88 \\
Instep height & 51.8 & 62.56 & 80.8 & \pm 4.29 \\
1st toe height & 7.6 & 16.92 & 27.7 & \pm 3.43 \\
Height of the external malleolus & 49.9 & 64.52 & 122.7 & \pm 7.93
\end{tabular}

The second basic acquisition step was designed to compile detailed information about the fitting of some shoes to the participant's feet. We purchased several pairs of 8 different shoe models that we thought representative of the models available in the market during the database acquisition (see top of Fig- 

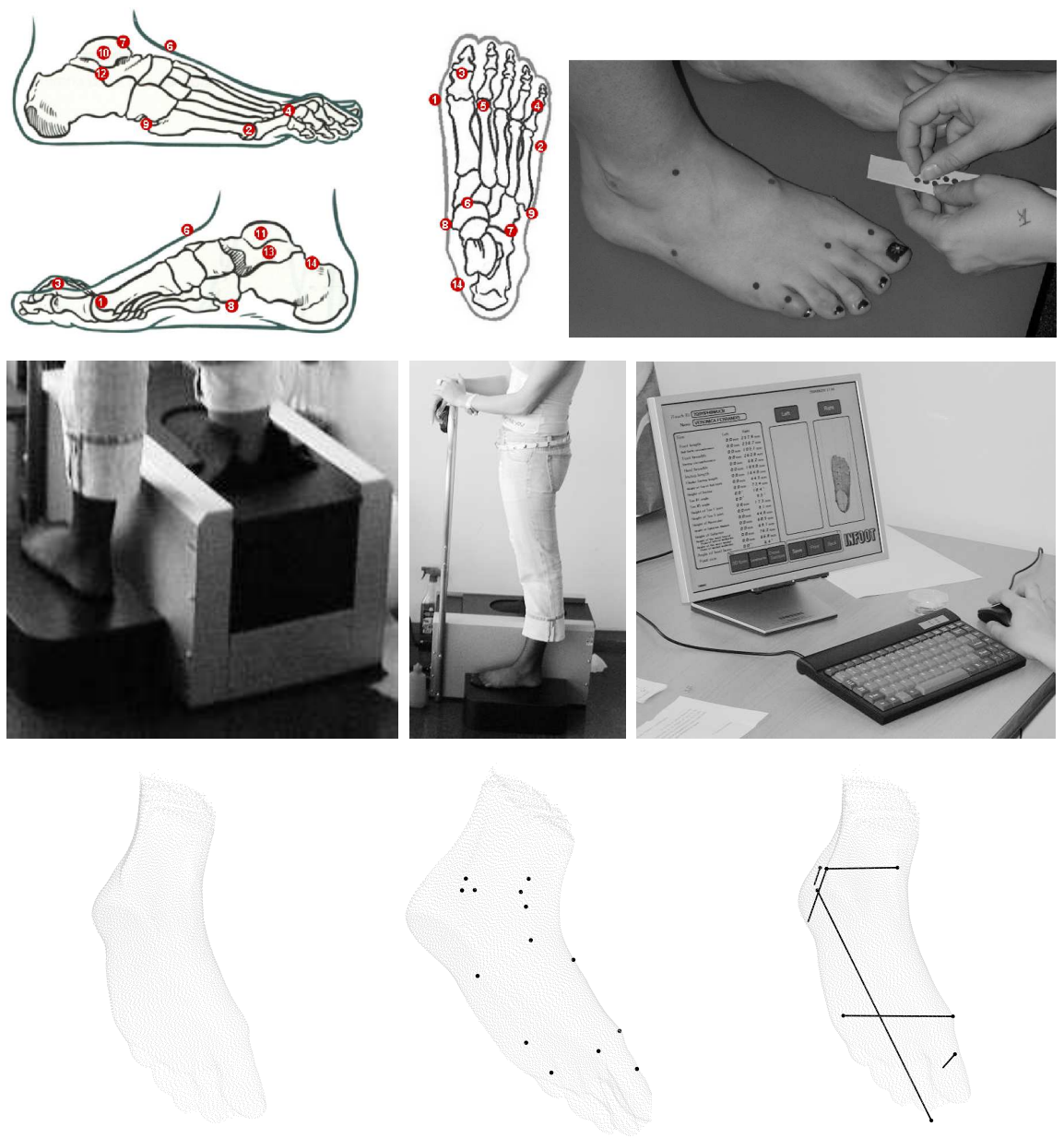

Fig. 1. Acquisition of 3D foot shapes. Top-left: locations of the 14 foot landmarks; 1) Metatarsal Tibiale, 2) Metatarsal Fibulare, 3) Highest point of the 1st toe at the interphalangeal joint, 4) Highest point of the 5th toe joint at the distal interphalangeal joint, 5) Head of the second metatarsal, 6) Instep point (Cuneiform), 7) Tentative junction point, 8) Navicular, 9) Tuberosity of five metatarsal, 10) The most lateral point of lateral malleolus, 11) The most medial point of medial malleolus, 12) Sphyrion Fibulare, 13) Sphyrion, and 14) Medial tentative heel upper point. Top-right: placement of adhesive markers at the locations of the 14 foot landmarks. Middle: scanner parts and participant's position and orientation during the scanning process. Bottom: output of the process; from left to right: 3D foot shape described as a cloud of points, locations of the 14 landmarks, and 6 podometric measurements derived from these landmarks (foot length, forefoot width, hell width, instep height, 1st toe height and height of the external malleolus). 
ure 2). Given a pair of shoes (and socks), the participant was asked to try it walking during 2 minutes and then fill in a questionnaire about the fitting in 15 different zones of the foot (see bottom of Figure 2). For each of these zones, the participant gave her perception of discomfort in a 4 -level ordinal scale $(0=$ none, $1=$ low, $2=$ medium, $3=$ high). Also, the participant answered questions about the global discomfort of the shoes and her general footwear preferences. This step lasted 5 minutes on average for the trial of each model. Unfortunately, the high time cost of the complete trial (40 minutes for 8 models) prevented trying all the available shoe models, so we decided to provide the participant with only 4 randomly selected models. Therefore, on average, each shoe model was tried by half of the 316 participants.
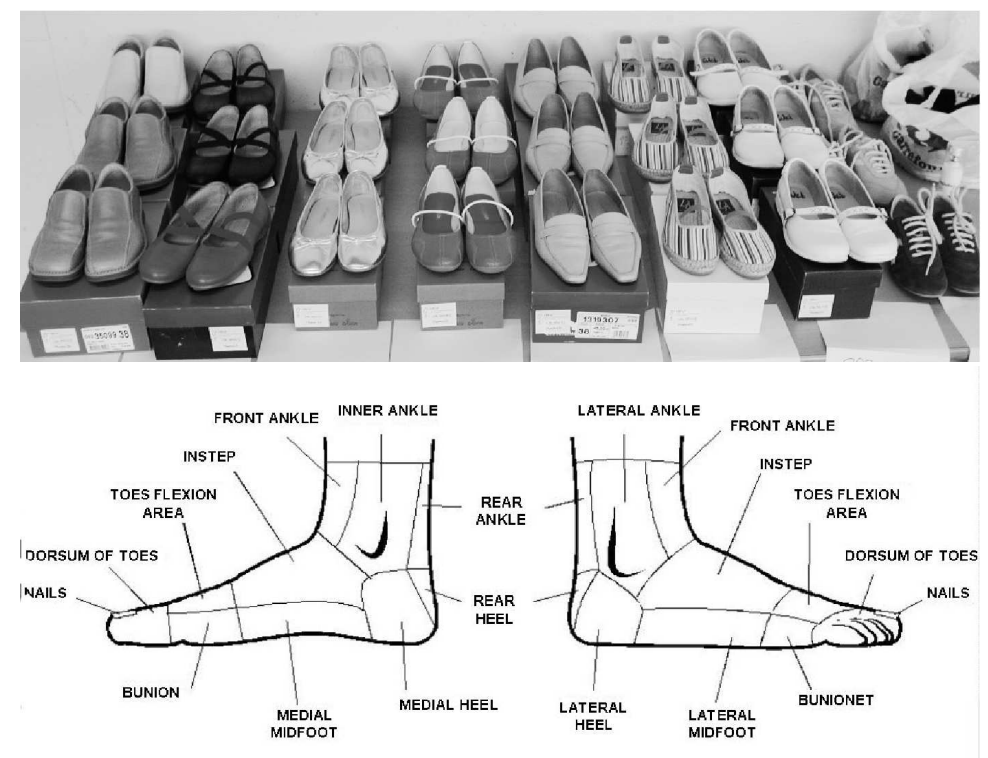

Fig. 2. Top: shoe models purchased. Bottom: zone division of the foot.

Table 2 shows the mean and the standard deviation of the discomfort variable for each shoe model and foot zone.

The MORFO3D foot database is available upon request for non-commercial use. The complete database also stores data of 100 women right foot of European shoe size 40. The data in the MORFO3D database is being used to study the relation between footwear fitting and podometric measurements. 
Table 2. Mean and standard deviation of the discomfort variable $(0=$ none, $1=$ low, $2=$ medium, $3=$ high) for each shoe model and foot zone.

\begin{tabular}{|c|c|c|c|c|c|c|c|c|c|c|}
\hline & \\
\hline & Foot zone & 1 & 2 & 3 & 4 & 5 & 6 & 7 & 8 & Average \\
\hline 1 & Rear heel & $0.3 \pm 0.7$ & $0.9 \pm 1.1$ & $0.3 \pm 0.7$ & $0.2 \pm 0.5$ & $0.5 \pm 0.8$ & $0.3 \pm 0.7$ & $0.5 \pm 0.8$ & $0.5 \pm 0.8$ & $0.5 \pm 0.8$ \\
\hline 2 & Lateral heel & $0.2 \pm 0.5$ & $0.7 \pm 1.0$ & $0.2 \pm 0.6$ & $0.1 \pm 0.4$ & $0.3 \pm 0.7$ & $0.2 \pm 0.6$ & $0.3 \pm 0.6$ & $0.3 \pm 0.7$ & $0.3 \pm 0.7$ \\
\hline 3 & Medial heel & $0.2 \pm 0.5$ & $0.7 \pm 1.0$ & $0.2 \pm 0.5$ & $0.1 \pm 0.3$ & $0.3 \pm 0.6$ & $0.2 \pm 0.5$ & $0.3 \pm 0.6$ & $0.3 \pm 0.6$ & $0.3 \pm 0.7$ \\
\hline 4 & Rear ankle & $0.3 \pm 0.6$ & $0.5 \pm 1.0$ & $0.2 \pm 0.6$ & $0.2 \pm 0.6$ & $0.3 \pm 0.7$ & $0.2 \pm 0.6$ & $0.4 \pm 0.8$ & $0.6 \pm 0.9$ & \\
\hline 5 & Lateral ankle & $0.2 \pm 0.5$ & $0.4 \pm 0.8$ & $0.1 \pm 0.4$ & $0.1 \pm 0.2$ & $0.1 \pm 0.5$ & $0.1 \pm 0.4$ & $0.2 \pm 0.5$ & $0.3 \pm 0.7$ & \pm 0.6 \\
\hline 6 & Inner ankle & $0.2 \pm 0.5$ & $0.3 \pm 0.8$ & $0.1 \pm 0.4$ & $0.1 \pm 0.2$ & $0.1 \pm 0.3$ & $0.1 \pm 0.4$ & $0.3 \pm 0.6$ & $0.4 \pm 0.7$ & \pm 0.5 \\
\hline 7 & Front ankle & $0.2 \pm 0.5$ & $0.3 \pm 0.7$ & $0.1 \pm 0.4$ & $0.1 \pm 0.4$ & $0.1 \pm 0.4$ & $0.1 \pm 0.4$ & $0.1 \pm 0.4$ & $0.4 \pm 0.8$ & \pm 0.5 \\
\hline 8 & Instep & $6 \pm 0.8$ & $1.4 \pm 1.1$ & $0.1 \pm 0.5$ & $0.5 \pm 0.8$ & $0.3 \pm 0.6$ & $0.2 \pm 0.5$ & $0.9 \pm 1.1$ & $0.8 \pm 0.9$ & $6 \pm 0.9$ \\
\hline & Lateral midfoot & $3 \pm 0.6$ & $0.9 \pm 1.0$ & $0.2 \pm 0.5$ & $0.2 \pm 0.5$ & $0.2 \pm 0.6$ & $0.1 \pm 0.4$ & $0.4 \pm 0.7$ & $0.5 \pm 0.8$ & \pm 0.7 \\
\hline & Media & $0.2 \pm 0$ & $0.8 \pm 1.0$ & $0.2 \pm 0.5$ & $0.2 \pm 0$ & & & & & \\
\hline 11 & Toes flexion area & $0.3 \pm 0.6$ & $1.3 \pm 1.1$ & $0.3 \pm 0.7$ & $0.2 \pm 0.5$ & $0.5 \pm 0.7$ & $0.2 \pm 0.5$ & $1.2 \pm 1.1$ & $0.5 \pm 0.8$ & $=0.9$ \\
\hline 12 & Bunionette & $0.2 \pm 0.5$ & $1.0 \pm 1.1$ & $0.3 \pm 0.6$ & $0.1 \pm 0.4$ & $0.3 \pm 0.7$ & 0.4 & & 0.7 & 0.7 \\
\hline 13 & Buni & $2 \pm 0.5$ & $0.9 \pm 1.1$ & $0.3 \pm 0.7$ & $0.1 \pm 0.4$ & $0.3 \pm 0.7$ & $0.2 \pm 0.5$ & $0.5 \pm 0.8$ & $0.3 \pm 0.6$ & $1 \pm 0.7$ \\
\hline 14 & Dorsum & $0.4 \pm 0.7$ & $1.4 \pm 1.2$ & $1.1 \pm 1.1$ & $0.2 \pm 0.5$ & $1.1 \pm 1.1$ & $0.5 \pm 0.8$ & $0.8 \pm 1.1$ & $0.5 \pm 0.8$ & \pm 1.0 \\
\hline 15 & Nails & $0.4 \pm 0.8$ & $1.2 \pm 1.2$ & $1.3 \pm 1.1$ & $0.2 \pm 0.5$ & $0.9 \pm 1.1$ & $0.7 \pm 0.9$ & $0.9 \pm 1.2$ & $0.5 \pm 0.8$ & $0.8 \pm 1.0$ \\
\hline & & $0.3 \pm 0.4$ & & $0.3 \pm 0.4$ & $0.2 \pm 0.3$ & $0.4 \pm 0.4$ & $0.2 \pm 0.3$ & $0.5 \pm 0.4$ & $0.5 \pm 0.4$ & $0.4 \pm 0.5$ \\
\hline
\end{tabular}

\section{$3 \quad$ Automatic detection of landmarks}

The classical method to detect foot anatomical landmarks described in section 2 requires an expert to manually place them. The landmark placement obtained from different experts, or from the same expert at different moments, can be different. In this work, we propose a technique to automatically perform this landmark placement.

The technique proposed is based on heuristics and takes into account the fact that the scanner places automatically the reference axis shown in the top-right of the Figure 3. It works initially with the complete 3D point clouds obtained from the scanner and can be described as follows. First, a set of initial landmarks is constructed from the complete cloud. It includes the landmarks shown as points $6,16,17$ and 21 in the top-left and top-center of the Figure 3. For instance, the landmark 17 is the point with a smaller $x$-coordinate from the complete $3 \mathrm{D}$ cloud and the landmark 21 is the point with $y$-coordinate $\simeq 0, z$-coordinate $\simeq 0$ and the greatest $x$-coordinate. The set of initial landmarks allows us to split the cloud along the $x$ and $z$-axes into three sub-clouds: upper, rear-lower and forelower subclouds. Then, more specic points are searched in each sub-cloud. For instance, in rear-lower subcloud, the points 19 and 20 from Figure 3 are located as those points with, respectively, the smallest and the greatest $y$ coordinate. Working in this way, the points $1,2,15$ and 18 are detected in the fore-lower subcloud, and the points $10-13$ in the upper subcloud. Note that most of the automatic landmarks are border points, that is, they are coordinate maximums or minimums in an axis of a sub-cloud. Only the landmarks 6, 12, 13 and 16 are not border points. The landmark 6 (instep point) is the intersection point of the cloud with the line that goes through the midpoint between landmarks 17 and 21 , in the direction of the $Z$ axis. The landmarks 12,13 and 16 are heuristically detected from, respectively, the landmarks 10, 11 and 17.

The set of landmarks detected automatically does not match exactly with that obtained manually (see Figure 1 ). Some landmarks that are located manually can be automatically detected, but not all of them. For instance, the land- 
mark points 8 and 9 of the top-left and top-center of Figure 3 show the joint point of two bones. Therefore, they can only be placed by touching the foot, since they can not be seen as any protuberance.

We have compared the landmark set obtained manually with the landmark set obtained using our automatic technique. As both sets do not completely match, we have compared their intersection. That is, given one foot, each automatic landmark from intersection points set has been compared with its respective manual landmark. We have use different approaches for it. On one hand, we have use three distance definitions: $L_{1}, L_{2}$ (or Euclidean Distance) and mean squared error $(M S E)$. The $L_{1}$ and $L_{2}$ distances from a manual landmark $x$ to its corresponding automatic landmark $y$ for a given foot are shown in the equations 1 and 2 . The results shown in Table 3 are the distance averages. The $M S E$ from the set of observed values (manual landmarks) $X=\left(x_{1}, x_{2}, \ldots, x_{N}\right)$ to calculated values (automatic landmarks) $Y=\left(y_{1}, y_{2}, \ldots, y_{N}\right)$ is given in the equation 3. The result is shown in Table 3.

$$
\begin{gathered}
L_{1}(x, y)=\left|x_{1}-y_{1}\right|+\left|x_{2}-y_{2}\right|+\left|x_{3}-y_{3}\right| \\
L_{2}(x, y)=\sqrt{\left(x_{1}-y_{1}\right)^{2}+\left(x_{2}-y_{2}\right)^{2}+\left(x_{3}-y_{3}\right)^{2}} \\
M S E(X, Y)=\sqrt{\frac{\sum_{i=1}^{N}\left(x_{i}-y_{i}\right)^{2}}{N}}
\end{gathered}
$$

On the other hand, Table 4 shows the error rate for different thresholds of tolerance. It is computed as follows: for each threshold value, we say that an automatic landmark matches with its corresponding manual landmark when the $L_{2}$ distance between them is smaller than the given threshold value (success); otherwise, we say that they do not match (error).

The bottom of Figure 3 shows two examples of application of our automatic technique. In the examples, the automatic landmarks are compared with the classical landmarks. In the left image, our technique works properly but, in the right one, it did not place the anatomical points in the correct position.

\begin{tabular}{|c|c|c|c|}
\hline Point & $L_{1}$ & $L_{2}$ & $M S E$ \\
\hline Lateral malleolus (10) & 33.68 & 22.39 & 5.8 \\
\hline External heel (19) & 60.63 & 46.18 & 7.78 \\
\hline Medial malleolus (11) & 43.77 & 30.02 & 6.62 \\
\hline Internal heel (20) & 41.89 & 29.80 & 6.47 \\
\hline Heel rear (17) & 18.15 & 14.01 & 4.26 \\
\hline Total & 39.62 & 28.48 & $\overline{6.19}$ \\
\hline
\end{tabular}

Table 3. Different error rates (in $\mathrm{mm}$ ) for each point of the intersection set and total error rate. Numbers in brackets correspond with those of the Figure 3. 

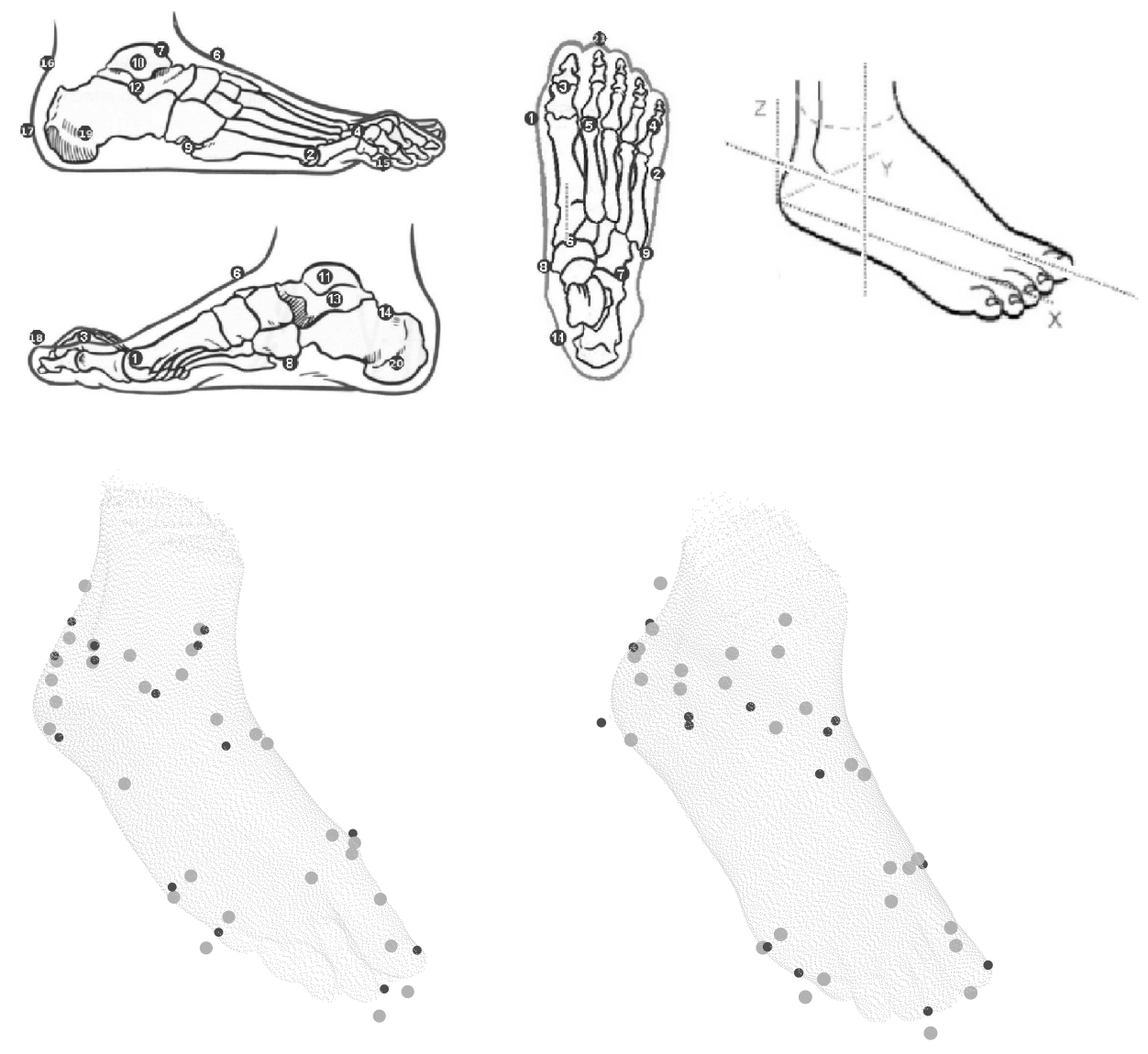

Fig. 3. Automatic detection of landmarks. Top-left and top-center: landmarks detected by the automatic method; 1) Metatarsal Tibiale, 2) Metatarsal Fibulare, 6) Instep point (Cuneiform), 10) The most lateral point of lateral malleolus, 11) The most medial point of medial malleolus, 12) Sphyrion Fibulare, 13) Sphyrion, 15) Most lateral point of the 5th toe, 16) Insertion of Achille's tendom in calcaneus, 17) Heel rearrest point, 18) Highest point of the 1st toe, 19) Most prominent point of the external heel, 20) Most prominent point of the internal heel and 21) Most advanced point of the 2nd toe. Landmarks numbered from 1 to 14 are those detected by the classical method (see Figure 1 for more details). Top-right: reference axis with lines that split the point clouds in three subclouds. Bottom : the automatic method application. Black points are manually placed by the expert, and grey points are detected automatically. In the left image the automatic method works properly, but in the right one the method fails 
Table 4. Error rate (\%) for different tolerance thresholds (distance in mm between classical and automatic anatomical points)

\begin{tabular}{l|rrrrrrr} 
Distance & 0 & 1 & 2 & 3 & 4 & 5 & 6 \\
Error & 98 & 90 & 76 & 66 & 60 & 56 & 52 \\
\hline Distance & 7 & 8 & 9 & 10 & 11 & 12 & 13 \\
Error & 49 & 47 & 44 & 42 & 40 & 38 & 36 \\
\hline Distance & 14 & 15 & 16 & 17 & 18 & 19 & 20 \\
Error & 34 & 33 & 32 & 31 & 30 & 29 & 28
\end{tabular}

Besides, the automatic landmark set of a foot can be used to compute a minimal but complete set of automatic podometry features, those shown in Table 1. These features can be used to automatically characterize the foot.

\section{Conclusions}

A foot database comprising 3D foot shapes and footwear fitting reports of more than 300 participants has been presented. It is called MORFO3D foot database. It can be used to study footwear fitting, and also to analyse anatomical features of the foot. In fact, we have presented a technique for automatic detection of foot anatomical landmarks that gives good results. The MORFO3D foot database is available upon request for non-commercial use.

\section{References}

1. I-ware laboratory. http://www.i-ware.co.jp/.

2. R. S. Goonetilleke and A. Luximon. Designing for comfort: a footwear application. In Proceedings of the Computer-Aided Ergonomics and Safety Conference'01, July 28-Aug 2 2001. Plenary session paper.

3. B. Nacher, E. Alcántara, S. Alemany, J. García-Hernández, and A. Juan. 3d foot digitalizing and its application to footwear fitting. In In Proc. of $3 D$ Modelling, 2004. 